\author{
Briefing Paper \\ ASHP Ambulatory Care Conference and Summit \\ March 3-4, 2014
}

\title{
Defining and Advancing Ambulatory Care Pharmacy Practice: It Is Time to Lengthen Our Stride
}

\author{
Dennis K. Helling and Samuel G. Johnson
}

Dennis K. Helling, Pharm.D., D.Sc., FCCP, FASHP, FAPhA, is Executive Director Emeritus, Pharmacy Operations and Therapeutics, Kaiser Permanente Colorado, and Clinical Professor, University of Colorado Skaggs School of Pharmacy and Pharmaceutical Sciences, Aurora (dennis.helling@gmail.com).

Samuel G. Johnson, Pharm.D., FCCP, BCPS (AQ-Cardiology), Clinical Pharmacy SpecialistApplied Pharmacogenomics, Kaiser Permanente Colorado; Clinical Assistant Professor, University of Colorado Skaggs School of Pharmacy and Pharmaceutical Sciences, Aurora; and Institute of Medicine Anniversary Fellow in Pharmacy, 2012 (sgjead@gmail.com).

\begin{abstract}
Purpose. This paper reviews the basic tenets of ambulatory care pharmacy practice, including (1) the historical development of patient-centered care provide by pharmacists, (2) the need for and value of comprehensive medication management, (3) the education, training, and qualifications of pharmacists, and (4) demonstrated improvement in health and health care outcomes from pharmacists' services.

Summary. When ambulatory care pharmacists engage in patient care to their full capacity, physician time is saved, access to care is improved, and clinical and economic outcomes are enhanced. There is a need for ambulatory care pharmacists to work toward optimizing safe medication use and optimizing medication therapy for patients with diabetes, asthma, cardiovascular disease, and renal disease. Other opportunities for the development of ambulatory care pharmacy services exist in preventive care, precision therapeutics, medication therapy management, mitigation of health care disparities, and in implementing national health care reform. Interprofessional patient care teams should include ambulatory care pharmacists in patient-centered medical homes and accountable care organizations. Ambulatory care pharmacy practice would benefit by enhancing specialty residency training and by creating a residency/fellowship for advanced subspecialty clinical practice and research. Provider status is essential to recognize pharmacists as an integral part of the patient care team.

Conclusion. By assertively advancing ambulatory care practice, pharmacy will help achieve the national priorities of improving patient care, patient health, and affordability of care.
\end{abstract}




\section{Introduction}

The Board of Pharmacy Specialties (BPS) offers the following definition for ambulatory care pharmacy practice, which was developed by a joint task force of the American Pharmacists Association (APhA), the American Society of Health-System Pharmacists (ASHP), and the American College of Clinical Pharmacy (ACCP):

Ambulatory care pharmacy practice is the provision of integrated, accessible health care services by pharmacists who are accountable for addressing medication needs, developing sustained partnerships with patients, and practicing in the context of family and community. This is accomplished through direct patient care and medication management for ambulatory patients, long-term relationships, coordination of care, patient advocacy, wellness and health promotion, triage and referral, and patient education and self-management. The ambulatory care pharmacists may work in both an institutional and community-based clinic involved in direct care of a diverse patient population. ${ }^{1}$

Several clear tenets frame the practice of ambulatory care pharmacy: (1) comprehensive medication management within integrated health systems, community pharmacies, and standalone clinical practices; (2) a growing work force of qualified and board-certified clinical pharmacists; (3) a growing emphasis on specialty and subspecialty training across ambulatory care practice settings; and (4) demonstrated improvement in health and care outcomes for patients in ambulatory care settings. Overall, this pharmaceutical care framework demonstrates a firm commitment from the profession to take accountability for health outcomes. ${ }^{2}$

\section{Historical successes since the $1960 \mathrm{~s}$}

The 1960s. Ambulatory care pharmacy practice sprang from humble beginnings.

Transformative changes in the pharmacy profession in the 1960s resulted in increased documentation of the economic and human benefits derived from optimizing medication use in the U.S. health system. Ultimately, this refocused efforts toward reducing medication-related morbidity and mortality, and shifted energy away from the product and transaction- 
based business of pharmacy toward patient-centered care.

One of the first documented examples of patient medication profile use within a community pharmacy practice setting was in 1960 in Berryville, Virginia. Eugene White was a pharmacist who transformed his drugstore into the first known office-based pharmacy practice. ${ }^{3}$

In 1962, Albert Ripley, a pharmacist at the Indian Hospital in Crow Agency, Montana, started filling patients' prescriptions based on medical record information instead of paper prescriptions. This was eventually adopted as a best practice by the Indian Health Service (IHS), which subsequently incorporated private consultation offices in most IHS facilities where pharmacists could assess patients and provide medication counseling. ${ }^{4}$

The 1970s. The 1970s saw these individual efforts lead to national action. In 1971, the National Center for Health Services Research and Development charged an interdisciplinary task force with development of standards for pharmacists' clinical practice. This effort, led by Donald Brodie, provided a tangible standard for clinical roles for pharmacists regardless of health care setting. ${ }^{5}$

In 1971, ASHP and the American Association of Colleges of Pharmacy (AACP) convened educators and practitioners from both institutional and community settings at an historic conference in Overland Park, Kansas, which discussed the "clinical aspects of pharmaceutical practice."

In 1973 at the University of Iowa, Helling and colleagues initiated the Iowa family practice-based clinical pharmacy program, representing one of the first examples of ambulatory care clinical pharmacy practice in family medicine. ${ }^{7,8}$

The 1980s. The 1980s showcased additional milestones in ambulatory care pharmacy practice, particularly within the Department of Veterans Affairs (VA). In 1987, Cheung et al. 
described a multi-institutional pharmacy system that was divided into inpatient and ambulatory care practices. Clinical services, research, and educational programs were integrated across settings to address the mission of providing quality services and support programs. ${ }^{9}$

The 1990s. The 1990s saw similar gains as the previous decades. In 1990, the Office of the Inspector General of the Department of Health and Human Services reviewed available clinical services in community pharmacy settings as part of a larger effort to reduce the incidence of adverse drug events (ADEs) among older adults. Sponsored by the Health Care Financing Administration (now known as the Centers for Medicare \& Medicaid Services, or CMS), the report found that "clinical pharmacy services add value to care for both institutionalized and ambulatory patients," and that "such value includes not only improvements in clinical outcomes and enhanced patient compliance, but also reductions in healthcare utilization costs associated with adverse drug reactions." 10

In 1994, an ASHP survey of federal hospital systems documented widespread ambulatory care pharmacy services, including pharmacist-managed drug clinics. Settings included Air Force, Navy, Public Health Service, and Department of Veterans Affairs hospitals. This was the first landscape survey of pharmacy in federal health systems, and it concluded that most settings offered comprehensive pharmacy systems, including clinical services as well as drug product distribution. ${ }^{11}$

In 1996, the Asheville Project was implemented in collaboration with the North Carolina Center for Pharmaceutical Care, the APhA Foundation, the North Carolina Pharmaceutial Association, and the city of Asheville, among others. Twelve community pharmacies contracted with the city of Asheville to provide diabetes management for city employees as part of a pilot project, with widely publicized success. ${ }^{12}$ 
In 1998, Carter and colleagues initiated a long-term study designed to assess the impact of ambulatory care clinical pharmacy services within selected VA medical centers. In the years that followed, multiple high-impact findings resulted from this work, including identification of risk factors for drug-related problems, development of a chronic disease indicator score, and estimates of the total clinical and economic impact of ambulatory care pharmacy services. ${ }^{13-16}$

In 1999, ASHP and APhA jointly developed standards and learning objectives for accreditation of community pharmacy residency training. This enabled accreditation of such programs through the ASHP Commission on Credentialing. ${ }^{17}$

The 2000s. Momentum for advancing ambulatory care pharmacy practice persisted in the new millennium. In 2006, the BPS promulgated a vision for a new specialty certification in ambulatory care pharmacy practice and commissioned a practice analysis to determine applicable domains, tasks, and knowledge that characterize the ambulatory care pharmacy specialist. Two years later this petition was approved by BPS, with the inaugural ambulatory care certification examination debuting in $2011 .^{18}$

\section{Areas of opportunity in ambulatory care pharmacy practice}

Improving clinical and economic outcomes. The above historical review of ambulatory care pharmacy practice serves as a foundation for discussing the current public health imperative to amplify pharmacist involvement in the care of ambulatory patients regardless of organizational or practice setting. It has been well documented that pharmacist-provided direct patient care significantly improves clinical and economic outcomes across health care settings, including ambulatory care. ${ }^{19}$

In their joint petition to BPS in 2008, leaders from ACCP, APhA, and ASHP 
highlighted continued opportunities for ambulatory care pharmacists to improve care for patients with chronic conditions, the costs of which exceeded $75 \%$ of the $\$ 2$ trillion U.S. annual health expenditures. ${ }^{20}$ Because medications — and medication management—play a critical role in the management of chronic diseases regardless of setting, the need for more ambulatory care clinical pharmacy services is quite evident. Moreover, such services reduce the potential for common medication-related problems, including prescribing and administration errors, nonadherence, and inappropriate use. Ambulatory care pharmacists are optimally positioned to provide comprehensive medication management for patients in a variety of ambulatory care settings, which is supported by published evidence of improved clinical and economic outcomes. ${ }^{21}$

Directly incorporating ambulatory care pharmacists into team-based care is part of the solution to the nation's health care crisis, with clear ability to improve patient care, population health, and affordability (i.e., the "Triple Aim"22). In fact, the Institute of Medicine (IOM) recognizes the critical opportunity for pharmacists to improve medication safety by facilitating comprehensive medication management. Per the IOM, "To close the gaps between best practice and usual care ... will require the collective expertise of a vast array of doctors, nurses, pharmacists, allied health professionals, social workers, and vested laypersons.,23

Several practical examples of clinical pharmacy services in the ambulatory care setting were implemented and validated at Kaiser Permanente Colorado (KPCO). A group-model integrated health system in the Denver-Boulder area, KPCO offered an ideal setting to optimize pharmacotherapy outcomes in a diverse, real-world patient population. One linchpin of the program was the culture of continuously establishing innovative pharmacy services and demonstrating their value (i.e., improved clinical and economic outcomes). ${ }^{24,25}$

Carter and colleagues at the University of Iowa conducted other compelling 
research. Their evaluation of blood pressure control and adherence to antihypertensives among ambulatory patients receiving collaborative care with physicians and clinical pharmacists demonstrated significant improvements in health outcomes. These findings further highlight important opportunities for ambulatory care clinical pharmacists to contribute to the primary care team. $^{26,27}$

While these examples focus on discrete areas of ambulatory care pharmacy practice, a recent systematic review of all published examples provided an estimate of the overall impact of ambulatory care pharmacy practice. Chisholm-Burns and colleagues reviewed articles that reported the impact of pharmacists in direct patient care across a variety of settings, approximately two-thirds of which were ambulatory care settings (including hospital-based clinics and community pharmacies). The majority of these interventions were classified as combination or multimodal, including those focused on improving patient understanding of medications or diseases, adherence interventions, drug utilization review (prospective and retrospective), and chronic disease management. ${ }^{28}$ Meta-analysis showed statistically significant improvements in clinical outcomes for hemoglobin A1C reduction, low-density lipoprotein (LDL) reduction, and systolic blood pressure reduction. In addition, improvements in humanistic outcomes were observed in medication adherence, patient knowledge, and patient-reported quality of life (i.e., general health).

A separate review of 126 studies by Chisholm-Burns et al. documented the impact of pharmacist interventions on economic outcomes. ${ }^{29}$ Most of the available studies were limited because of incomplete or partial analyses or methodological shortcomings. Nearly $50 \%$ of the studies included ambulatory care practices. As in the previous example, most $(\sim 56 \%)$ of the pharmacist interventions were classified as multimodal. Twenty of 126 studies reported positive 
economic benefits. More well-designed trials are required to definitively assess the economic impact of pharmacist clinical interventions among ambulatory patients.

Another evaluation of the economic impact of clinical pharmacy services by Perez and colleagues in 2007 included 93 articles that documented outcomes from services in different settings. ${ }^{30}$ Approximately $40 \%$ of the articles described services performed in ambulatory care clinics or community settings, and the most frequently provided services were pharmacotherapeutic monitoring, target drug programs, or disease state management. The authors demonstrated that for every dollar invested in clinical pharmacy service, $\$ 4.81$ of savings were incurred in reduced costs or other return on investment. While methodological limitations exist in these studies, the overall findings support the notion that clinical pharmacy services provide significant economic benefit as well as clinical benefit.

Optimizing safe medication use. While the studies highlighted above certainly bolster the profession, much effort remains in order to realize the full potential of ambulatory care pharmacy practice. Preventable medication errors are still too prevalent and often result in patient injury and added costs, which has prompted several national initiatives from the IOM. A recent report estimated national annual costs associated with preventable ADEs in the ambulatory care setting at $\$ 887$ million, equating roughly to $\$ 1,983$ per $\mathrm{ADE} .{ }^{31} \mathrm{~A}$ study by Raebel and colleagues at KPCO provided evidence supporting an ambulatory care pharmacist intervention to reduce inappropriate prescribing in elderly patients (65 or older) using a computerized medication safety alert. ${ }^{32}$ Gandhi et al. found that ADEs occurred in roughly $27 \%$ of patients in the ambulatory care setting (i.e., four times higher than that estimated for hospitalized patients), of which $39 \%$ were deemed preventable. ${ }^{33}$ Furthermore, nearly two-thirds of the preventable ADEs were reported by patients as ADE-related symptoms to physicians but 
not acted upon during the three-month study period. Collectively, these reports point to the need and value of expanded ambulatory care pharmacy practices.

Optimizing diabetes care. As of 2011, an estimated 18.8 million patients of all ages were diagnosed with diabetes mellitus (DM); moreover, the incidence of DM and related complications is increasing. ${ }^{34}$ Despite widespread availability of affordable evidence-based pharmacotherapy, many patients with DM still do not receive therapies that are clearly indicated. Ambulatory care pharmacists have used population-based strategies as well as case management to address this therapeutic gap. . $^{35,36}$

Optimizing asthma care. Asthma affects an estimated 25 million people (approximately 1 in 12) in the United States. ${ }^{37}$ In 2010 , there were 15.5 million outpatient asthma-related visits to physicians' offices and hospital outpatient departments and 1.8 million visits to emergency departments. Without effective management, acute exacerbations are more frequent, and these require urgent treatment and result in $\$ 56$ billion in medical costs, lost school and work days, and premature deaths according to 2007 data. ${ }^{37}$ An Asheville project (apart from the project on management of diabetes) demonstrated an association between pharmacist-provided asthma education and long-term medication management services with improved clinical outcomes and reduced overall costs of care (despite increased cost of medications). ${ }^{38}$ This report supports expanded roles for ambulatory care pharmacists to assist patients in the use of asthma medications and devices.

Optimizing cardiovascular disease care. Cardiovascular disease remains the leading cause of death in the United States with approximately 600,000 people perishing from heart disease (roughly 1 in 4 deaths). The economic burden of coronary heart disease in the U.S. is $\$ 108$ billion annually, including costs of health care services, medications, and lost 
productivity. ${ }^{39}$ Because medications are a mainstay of treatment for hypertension (HTN), dyslipidemia, arrhythmias, angina, heart failure, thromboembolism, and primary or secondary prevention of cardiovascular disease (e.g., coronary artery disease, myocardial infarction, and stroke), ambulatory care clinical pharmacy specialists have important opportunities to assist patients in achieving optimal medication and health outcomes, including reduced mortality related to cardiovascular disease, antithrombotic-related hemorrhage, and thromboembolism. ${ }^{40,41}$

Optimizing renal disease care. The primary causes of chronic renal disease are HTN and diabetes, based on 2006 data. $^{42}$ More than 20 million Americans have chronic renal disease, with at least as many at increased risk for developing the disease. ${ }^{42}$ Adherence to complex medication regimens is crucial to stabilizing renal disease processes and slowing the progression of renal disease and comorbidities. Ambulatory care pharmacist specialists contribute significantly to prevention and management of chronic renal disease and end-stage renal disease through both case-based ${ }^{43}$ and population-based ${ }^{44}$ approaches.

Optimizing preventive care. As cited in the Healthy People 2020 report, ${ }^{45}$ preventive clinical interventions such as immunizations and tobacco cessation improve economic and health outcomes. Among the national health objectives expressed in Healthy People 2020 is "prevent disease, disability, and death from infectious diseases, including vaccine-preventable diseases." ${ }^{45}$ Ambulatory care pharmacists are now routinely acquiring specialized knowledge and training to screen patients and administer vaccines in nearly all states and across a variety of settings. ${ }^{46,47}$ Approximately 50 million adults in the United States smoke cigarettes, which ultimately will result in disability and premature death for half of them. For individuals who quit smoking, the risk of tobacco-related morbidity declines and eventually is similar to that of a nonsmoker, which reinforces the importance of tobacco cessation efforts. ${ }^{48}$ Ambulatory care pharmacists provide 
access and care for patients and are appropriately positioned to implement evidence-based tobacco cessation treatments. ${ }^{49,50}$

Implementing precision therapeutics. Implementation of clinically relevant pharmacogenetic testing has been widely touted as the next frontier for individualizing pharmacotherapy for patients. ${ }^{51,52}$ Despite compelling models demonstrating the potential impact of pre-emptive genotyping on medication-related and economic outcomes, examples of their successful application in noninstitutional ambulatory care pharmacy settings are few. O'Connor and colleagues demonstrated the feasibility of implementing a pharmacogenetic testing service within a chain community pharmacy. ${ }^{53}$

Expanding medication therapy management. It remains critically important to promote the engagement of ambulatory care pharmacists in medication therapy management (MTM) processes for Medicare and Medicaid beneficiaries. Indeed, the benefits of MTM are integral to community pharmacy practice as well as to ambulatory care clinical practice. Studies of the impact of ambulatory care pharmacist MTM services demonstrate improved care coordination, reduced medication-related problems, and associated mortality improvements. ${ }^{54,55}$ In addition, development of collaborative drug therapy management (CDTM) protocols, clinical practice guidelines, and thoughtful medication formularies will further facilitate an expansion of MTM to more ambulatory care health settings. Finally, pioneering work in establishment of MTM Current Procedural Terminology (CPT) payment codes paves the way for reimbursement of MTM services. ${ }^{56}$

Mitigating health care disparities. Significant health care disparities result from limited access to care, further highlighting the need for expanded ambulatory care pharmacy services. The reports highlighted above demonstrate the ability of ambulatory care pharmacists to engage 
directly with patients and improve health care access. Specific cases of ambulatory care pharmacists mitigating health care disparities include reports by the University of Southern California School of Pharmacy ${ }^{57}$ and the El Rio Health Center in Tucson, Arizona. ${ }^{58}$

\section{Opportunities created by national health care reform}

In response to serious national health care problems related to access, cost, and quality, health care leaders are pursuing solutions, especially in chronic disease management, prevention, and optimal use of medications,${ }^{59}$ creating more opportunities for ambulatory care pharmacists. An important facet of health care reform is a shift in payment structure from fee-for-service to pay-for-outcomes (or bundled, value-based payment). National and state governments and employer groups are fostering payment models that incentivize improved outcomes, lowed costs, increased access to care. Embodied within these objectives are greater accountability among providers and patients and implementation of innovative health care and technology solutions. Patient care by ambulatory care pharmacists is aligned with a pay-for-outcomes model, as demonstrated by the evidence of the value of pharmacist interventions that increase quality of care and decrease health care costs.

\section{Imperative for interprofessional team-based care}

There is a vital need to continue to establish high-performance interprofessional teams that include ambulatory care pharmacists within accountable care organizations (ACOs) and patient-centered medical homes (PCMHs). ${ }^{59}$ The changes needed to shift ambulatory care away from physician-centered care delivery toward interprofessional team-based care will not happen overnight. While interprofessional team-based care is central to patient-centered care, it is unreasonable to expect health professionals with different training, perspectives, and experiences to simply coalesce without a concerted multilevel initiative. 
Shifting from "incidental" teamwork exposure to "deliberate and strategic" training as interprofessional teams, at both undergraduate and postgraduate levels, is imperative. It is essential to continue building and refining interprofessional programs in undergraduate and postgraduate pharmacy education to prepare practitioners who are "team-ready" to focus on optimizing patient care in ambulatory care settings.

\section{Assisting health care providers through expanded scope of practice}

Figure 1 illustrates how the time demands on primary care physicians exceed the available hours. ${ }^{60}$ Caring for 2,500 patients with the five most common chronic conditions would require a physician who works alone 6.7 hours per day. An additional 7.4 hours per day would be required to provide the panel of patients with important preventive services, especially those strongly recommended by the U.S. Preventive Services Task Force at Level A or B evidence. ${ }^{61}$ Added to that would be 4.6 hours required to care for acute problems. Hence, to adequately care

Figure 1. Time demands on primary care physicians for 2,500 patients. ${ }^{60}$

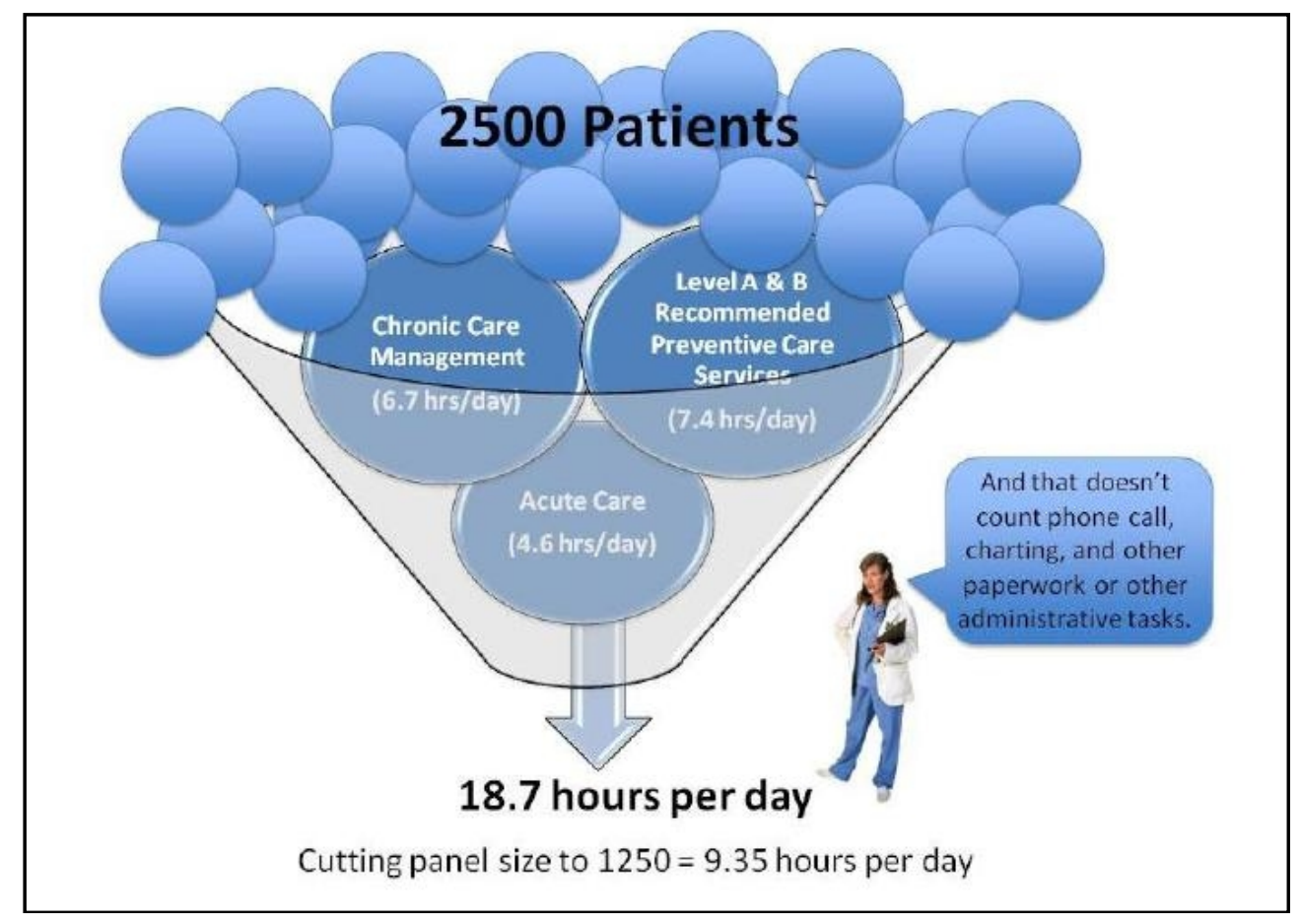


for a panel size of 2,500 patients, a primary care physician must work 18.7 hours a day, not including time required for phone calls, medication refills, charting, and other administrative tasks.

A number of projects have demonstrated that many patient visits to primary care providers, especially for chronic disease management, involve issues that could be handled by ambulatory care pharmacists via CDTM protocols. ${ }^{62}$ Many patient care and care-coordination activities are more optimally provided by pharmacist members of a care team. ${ }^{63}$ Consider the following examples:

- Most physicians deliver only 55\% of recommended care, and $42 \%$ report not having enough time with their patients. ${ }^{64}$

- Development of clinical pathways within ambulatory care pharmacies that streamline approval of medication refills by CDTM protocol frees up physician time for direct patient care. $^{65}$

- Providers spend $13 \%$ of their day on care coordination administrative activities and only half of their time on activities using their medical knowledge. Use of coordinated telepharmacy services that provide MTM and medication reconciliations during transitions of care effectively reduces this burden. ${ }^{66}$

These examples indicate how ambulatory care pharmacists who practice at the top of their license can save physician time, optimize physician skills, improve access to care, and enhance clinical and economic outcomes.

\section{Defining the scope of ambulatory care pharmacy practice}

Providing comprehensive medication management for patients is a primary responsibility of ambulatory care pharmacists. This responsibility becomes even more important when considering the medication-related problems of the growing U.S. elderly population and the trend toward managing more acute conditions and acute exacerbations of chronic conditions in ambulatory care settings.

Physicians are increasingly drawn into specialty and subspecialty practices rather 
than primary care ${ }^{67}$ There is already a dearth of primary care physicians in the United States, and the prospect of expanding the shortage troubles many health care leaders. Ambulatory care pharmacists have an opportunity to offset some of the anticipated deficit in the primary care work force. Parallel with the imperative for pharmacists to transition into a larger role in ambulatory care is the need for updated competency and practice standards tied to existing credentialing and certification pathways. As discussed in the next section of this paper, the current system of postgraduate pharmacy education provides a good foundation for enhancing the preparation of ambulatory care pharmacists for both generalist and specialist practice.

In the community pharmacy setting, ambulatory care clinical pharmacists can expand their practices to include a wide range of patient services, including immunizations and travel medicine, MTM, patient counseling and education, medication monitoring (e.g., such as for medications that have a Risk Evaluation and Mitigation Strategy), and selective chronic disease management under CDTM (e.g., hypertension, diabetes, primary coronary heart disease prevention, smoking cessation, asthma, high-risk medication monitoring, medication refill, and adherence programs).

In the clinic setting, ambulatory care clinical pharmacists can leverage CDTM protocols/clinical pathways to provide comprehensive medication management, transitions of care, direct clinical services, anticoagulation and anemia management, and management of other chronic diseases (e.g., secondary coronary heart disease prevention, depression, diabetes, hypertension, asthma, chronic kidney disease, chronic obstructive pulmonary disease [COPD], human immunodeficiency virus [HIV], and hepatitis C virus [HCV]). Additionally, they can also assist in drug therapy management of complex acute conditions (e.g., complicated urinary tract infections, seizures, pain management) and acute exacerbations of chronic diseases 
(e.g., heart failure, asthma, COPD).

Finally, greater collaboration between ambulatory care pharmacists in community settings and those in clinic settings is an opportunity to improve access to clinical pharmacy expertise. Preliminary research indicates that establishment of statewide practice networks for community-based and clinic-based ambulatory care pharmacists allows for efficient leveraging of resources and expertise across practice settings, which may be especially important in rural areas or for patients with otherwise unmet needs. ${ }^{68}$ Some examples include patients in rural or remote settings with complex conditions such as $\mathrm{HIV}, \mathrm{HCV}$, heart failure, or cancer.

\section{Advancing specialty and subspecialty training in ambulatory care pharmacy practice}

Advancing the current model of postgraduate training offers potential to address opportunities in chronic disease management and ambulatory care clinical pharmacy practice. In order to produce ambulatory care pharmacy practitioners, who, like physicians, are trained in specialties and subspecialties, advanced formalized training is required. In a future-oriented presentation at the 2013 ASHP National Pharmacy Preceptors Conference, we proposed that postgraduate year-one (PGY1) residency training should provide broad-based entry-level exposure to ambulatory care pharmacy operations, clinical services, and research. In our proposal, the postgraduate year-two (PGY2) ambulatory care specialty residency would focus on training and research experience in internal medicine or family practice. Further, we proposed that a new postgraduate year-three (PGY3) residency/fellowship could provide advanced subspecialty training and clinical research in an ambulatory care subspecialty clinical area. (For example, in endocrinology, this could be a sustainable training and practice pathway for caring for ambulatory patients with diabetes.) This model could also apply to cardiology and oncology. Further, to meet the pharmacy leadership needs of larger multihospital health systems, there is an 
opportunity to augment PGY2 specialty ambulatory care residencies with a new PGY3-type residency/fellowship in advanced clinical administrative leadership, which could include advanced business management and leadership training along with optional concurrent graduate coursework (e.g., MBA or MPH).

\section{Provider status for ambulatory care pharmacists}

The earnest pursuit of provider status has the potential to substantially advance ambulatory care pharmacy practice. Many pharmacy practice leaders believe that provider status is essential for pharmacists to become an integral part of patient care teams.

Many proponents of pharmacist provider status believe that pharmacists should be recognized for their value in improving patient outcomes in all practice settings. It is also argued that provider status recognition is consistent with substantial advancement in credentialing and certification of pharmacists providing direct patient care, increased scope of practice recognized in nearly all states, and advances in the standards for accredited Doctor of Pharmacy education and accredited postgraduate residency training.

Momentum for provider status is gathering at the grassroots level as evidenced by recent legislation passed in California. A new law authorizes all licensed pharmacists to administer drugs and biologics by injection when ordered by a prescriber; provide consultation, training, and education about drug therapy, disease management, and disease prevention; and order and interpret tests to monitor and manage the efficacy and toxicity of drug therapies in coordination with the patient's prescriber. The law also establishes an advanced practice pharmacist (APP) recognition and authorizes APPs to perform patient assessments; order and interpret drug therapy-related tests in coordination with the patient's prescriber; refer patients to other health care providers; initiate, adjust, and discontinue drug therapy with an order by the patient's 
prescriber and according to established protocols; and participate in the evaluation and management of diseases and health conditions in collaboration with other health care providers. Pharmacists seeking recognition as APPs are required to complete two of three criteria (certification in a relevant area of practice; a postgraduate residency program; one year of experience providing clinical services to patients under a collaborative practice agreement or protocol with a physician, APP pharmacist, CDTM pharmacist, or health system) ${ }^{69,70}$ Several other states, including Colorado, New Mexico, and North Carolina, have similar provisions for pharmacist provider status. In addition, a number of state Medicaid programs authorize pharmacist reimbursement for Medicaid MTM services. ${ }^{71,72}$

Now is the time for the pharmacy profession to "lean into provider status, to establish a credentialing process in all pharmacy environments so we can make direct patient care services essential health care services for all our patients., ${ }^{, 73}$

\section{Conclusion}

"If you build it, he will come." In the popular 1989 movie Field of Dreams, a cornfield counseled character Ray Kinsella (played by Kevin Costner) to build a baseball diamond on his farm. In some ways the pharmacy profession faces a similar premise: Our dream will not come true unless we build it.

Ambulatory care pharmacists serve their patients, community, and society through a harmonious array of functions. These functions begin and end with competent, compassionate, patient-centered care provided by community pharmacists, institutional-based ambulatory care pharmacists, and ambulatory care clinical pharmacy specialists. Depending on the needs of the patient and the resources required, the ambulatory care pharmacist provides medication management as an integral part of an interprofessional team-based care symphony. 
This is a crucible moment for the pharmacy profession. To meet the test of this moment, we must seek out higher levels of certification for our practice sites and ourselves. We must embrace documentation in a way that speaks the language of other health care providers and payers. We must continue to demonstrate our value on nationally recognized metrics, as these are the basis for how other health care providers and health systems are rated, compared, and paid. This is the way we will optimally assist physicians and health systems to earn support for provider status and payment for patient care services. Now is the time for ambulatory care pharmacy to lengthen its stride ${ }^{74}$ and remember the wisdom of legendary coach John Wooden:

"Don't measure yourself by what you have accomplished, but what you should have accomplished with your ability."

\section{References}

1. Board of Pharmacy Specialties. Specialties - ambulatory care. http://www.bpsweb.org/specialties/AmbulatoryCarePharmacy.cfm (accessed 2013 Dec 2).

2. Hepler CD, Strand LM, Tromp D et al. Critically examining pharmaceutical care. $J$ Am Pharm Assoc. 2002;42(Suppl 1):S18-9.

3. White EV, Latif DA. Office-based pharmacy practice: past, present, and future. Ann Pharmacother. 2006;40:1409-14.

4. Church RM. Pharmacy practice in the Indian Health Service. Am J Hosp Pharm. 1987; 44:771-5.

5. Anon. Report of task force on the pharmacist's clinical role. J Am Pharm Assoc. 1971; 11:482-5.

6. Lachner BJ. Problems and concerns in implementation of clinical pharmacy programs. A hospital administrator. Am J Hosp Pharm. 1971;28:882-5.

7. Brown DJ, Helling DK, Jones ME. Evaluation of clinical pharmacist consultations in a family practice office. Am J Hosp Pharm. 1979;36:912-5.

8. Helling DK. Family practice pharmacy service: Part I. Drug Intell Clin Pharm. 1981; 15:9717.

9. Cheung A, Sleight SM, Sisson NM. Pharmacy practice in the Veterans Administration. Am J Hosp Pharm. 1987;44:747-54.

10. Office of the Inspector General, DHHS. The clinical role of the community pharmacist. Report OEI-89-89160, November 1990. http://oig.hhs.gov/oei/reports/oei-01-89-89160.pdf (accessed 2013 Sep 20).

11. Crawford SY, Santell JP. ASHP national survey of pharmaceutical services in federal hospitals—1993. Am J Hosp Pharm. 1994;51:2377-93. 
12. Cranor CW, Bunting BA, Christensen DB. The Asheville Project: long-term clinical and economic outcomes of a community pharmacy diabetes care program. J Am Pharm Assoc. 2003;43:173-84.

13. Carter BL, Malone DC, Valuck RJ et al. The IMPROVE study: background and study design. Impact of managed pharmaceutical care on resource utilization and outcomes in Veterans Affairs medical centers. Am J Health-Syst Pharm. 1998;55:62-7.

14. Isaksen SF, Jonassen J, Malone DC et al. Estimating risk factors for patients with potential drug-related problems using electronic pharmacy data. Ann Pharmacother. 1999;33:406-12.

15. Malone DC, Billups SJ, Valuck RJ et al. Development of a chronic disease indicator score using a Veterans Affairs medical center medication database. J Clin Epidemiol. 1999;52:5517.

16. Malone DC, Carter BL, Billups SJ et al. An economic analysis of a randomized, controlled, multicenter study of clinical pharmacist interventions for high-risk veterans: the IMPROVE study. Impact of managed pharmaceutical care resource utilization and outcomes in Veterans Affairs medical centers. Pharmacotherapy. 2000;20:1149-58.

17. Unterwagner WL, Zeolla MM, Burns AL. Training experiences of current and former community pharmacy residents, 1986-2000. J Am Pharm Assoc. 2003;43:201-6.

18. Board of Pharmacy Specialties (BPS) News Release 2009-05. June 15, 2009.

19. Giberson S, Yoder S, Lee MP. Improving patient and health system outcomes through advanced pharmacy practice. A report to the U.S. Surgeon General. Office of the Chief Pharmacist. U.S. Public Health Service. Dec 2011. http://www.accp.com/docs/positions/misc/Improving_Patient_and_Health_System_Outcome s.pdf (accessed 2013 Dec 2).

20. Webb E. Specialization and specialties in pharmacy practice: "gradual evolution or intelligent design?" ACCP Rept. 2008;27(Apr):3-5. http://www.accp.com/docs/report/0408.pdf (accessed 2013 Dec 2).

21. Giberson SF. Transform health care: a call to action for pharmacy. Am J Health-Syst Pharm. 2013;70:1601-4.

22. Institute for Healthcare Improvement. IHI triple aim initiative. http://www.ihi.org/offerings/Initiatives/TripleAIM/Pages/default.aspx (accessed 2013 Dec 2).

23. Adams K, Corrigan JM, eds. Priority areas for national action. Transforming health care quality. Washington, DC: The National Academies Press; 2003.

24. Helling DK, Nelson KM. How to develop and implement a program to continuously demonstrate the value of clinical pharmacy services. Pharmacotherapy. 2000;20(10 Pt 2):340S-344S.

25. Helling DK, Nelson KM, Ramirez JE et al. Kaiser Permanente Colorado region pharmacy department: innovative leader in pharmacy practice. J Am Pharm Assoc. 2006;46:67-76.

26. Carter BL, Ardery G, Dawson JD et al. Physician and pharmacist collaboration to improve blood pressure control. Arch Intern Med. 2009;169:1996-2002.

27. Weber CA, Ernst ME, Sezate GS et al. Pharmacist-physician comanagement of hypertension and reduction in 24-hour ambulatory blood pressures. Arch Intern Med. 2010;170:1634-9.

28. Chisholm-Burns MA, Kim Lee J, Spivey CA et al. U.S. pharmacists' effect as team members on patient care: systematic review and meta-analyses. Med Care. 2010;48:923-33. 
29. Chisholm-Burns MA, Graff Zivin JS, Lee JK et al. Economic effects of pharmacists on health outcomes in the United States: a systematic review. Am J Health-Syst Pharm. 2010; 67:1624-34.

30. Perez A, Doloresco F, Hoffman JM et al. Economic evaluations of clinical pharmacy services: 2001-2005. Pharmacotherapy. 2009;29:128.

31. Aspden P, Wolcott J, Bootman JL, et al., eds. Preventing medication errors: quality chasm series. Washington, DC: National Academies Press, 2007.

32. Raebel MA, Charles J, Dugan J, et al. Randomized trial to improve prescribing safety in ambulatory elderly patients. J Am Geriatr Soc. 2007;5:977-85.

33. Gandhi TK, Weingart SN, Borus J et al. Adverse drug events in ambulatory care. $N$ Engl $J$ Med. 2003;348:1556-64.

34. American Diabetes Association. Diabetes statistics. http://www.diabetes.org/diabetesbasics/diabetes-statistics/?loc=DropDownDB-stats (accessed 2013 Sep 27).

35. Tran MT, Billups SJ. Can guideline adherence be improved by letter outreach? Diabetes Care. 2008;31:e22.

36. Brummel AR, Soliman AM, Carlson AM et al. Optimal diabetes care outcomes following face-to-face medication therapy management services. Popul Health Manag. 2013;16:28-34.

37. Centers for Disease Control and Prevention. Asthma FastStats.

http://www.diabetes.org/diabetes-basics/diabetes-statistics/?loc=DropDownDB-stats (accessed 2013 Sep 27).

38. Bunting BA, Cranor CW. The Asheville project: long-term clinical, humanistic, and economic outcomes of a community-based medication therapy management program for asthma. J Am Pharm Assoc. 2006;46:133-47.

39. Centers for Disease Control and Prevention. Heart disease facts. http://www.cdc.gov/heartdisease/facts.htm (accessed 2013 Sep 27).

40. Merenich JA, Olson KL, Delate T et al. Mortality reduction benefits of a comprehensive cardiac care program for patients with occlusive coronary artery disease. Pharmacotherapy. 2007;27:1370-8.

41. Witt DM, Sadler MA, Shanahan RL et al. Effect of a centralized clinical pharmacy anticoagulation service on the outcomes of anticoagulation therapy. Chest. 2005;127:151522.

42. National Kidney and Urologic Diseases Information Clearinghouse. Kidney disease statistics for the United States. http://kidney.niddk.nih.gov/KUDiseases/pubs/kustats/index.aspx (accessed 2013 Sep 27).

43. Pai AB, Boyd A, Depczynski J et al. Reduced drug use and hospitalization rates in patients undergoing hemodialysis who received pharmaceutical care: a 2-year, randomized, controlled study. Pharmacotherapy. 2009;29:1433-40.

44. Bhardwaja B, Carroll NM, Raebel MA et al. Improving prescribing safety in patients with renal insufficiency in the ambulatory setting: the drug renal alert pharmacy (DRAP) program. Pharmacotherapy. 2011;31:346-56.

45. Healthy People 2020. Topics and objectives. http://www.healthypeople.gov/2020/topicsobjectives2020/default.aspx (accessed 2013 Sep 27).

46. Goad JA, Taitel MS, Fensterheim LE et al. Vaccinations administered during off-clinic hours at a national community pharmacy: implications for increasing patient access and convenience. Ann Fam Med. 2013;11:429-36. 
47. Anon. Pharmacist-provided immunization compensation and recognition: white paper summarizing APhA/AMCP stakeholder meeting. J Am Pharm Assoc. 2011; 51:704-12.

48. Centers for Disease Control and Prevention. Cigarette smoking in the United States. Current cigarette smoking among U.S. adults aged 18 years and older.

http://www.cdc.gov/tobacco/campaign/tips/resources/data/cigarette-smoking-in-unitedstates.html (accessed 2013 Sep 27).

49. Zillich AJ, Corelli RL, Zbikowski SM et al. A randomized trial evaluating 2 approaches for promoting pharmacy-based referrals to the tobacco quitline: methods and baseline findings. Res Social Adm Pharm. 2013;9:27-36.

50. Bauld L, Boyd KA, Briggs AH et al. One-year outcomes and a cost-effectiveness analysis for smokers accessing group-based and pharmacy-led cessation services. Nicotine Tob Res. 2011;13:135-45.

51. Schildcrout JS, Denny JC, Bowton E et al. Optimizing drug outcomes through pharmacogenetics: a case for preemptive genotyping. Clin Pharmacol Ther. 2012;92:235-42.

52. Crews KR, Cross SJ, McCormick JN et al. Development and implementation of a pharmacist-managed clinical pharmacogenetics service. Am J Health-Syst Pharm. 2011;68:143-50.

53. O'Connor SK, Ferreri SP, Michaels NM et al. Making pharmacogenetic testing a reality in a community pharmacy. J Am Pharm Assoc. 2012;52:e259-65.

54. Welch EK, Delate T, Chester EA et al. Assessment of the impact of medication therapy management delivered to home-based Medicare beneficiaries. Ann Pharmacother. 2009;43:603-10.

55. Anon. Finding a national audience for MTM. http://www.pharmacist.com/finding-nationalaudience-mtm (accessed 2013 Oct 7).

56. Isetts BJ, Buffington DE. CPT code-change proposal: national data on pharmacists' medication therapy management services. Consult Pharm. 2007;22:684-9.

57. Leal S. Medications, rationing, and health care: the role of pharmacists in bridging the gap. $J$ Health Care Poor Underserved. 2005;16:418-20.

58. Anon. Health care costs to reach nearly one-fifth of GDP by 2021. http://www.kaiserhealthnews.org/daily-reports/2012/june/13/health-care-costs.aspx (accessed 2013 Sep 27).

59. Abrons JP, Smith M. Patient-centered medical homes: primer for pharmacists. J Am Pharm Assoc. 2011;51:e38-48.

60. Østbye T, Yarnall KS, Krause KM et al. Is there time for management of patients with chronic diseases in primary care? Ann Fam Med. 2005;3:209-14.

61. Pollak KI, Krause KM, Yarnall KS et al. Estimated time spent on preventive services by primary care physicians. BMC Health Serv Res.2008;8:245.

62. Heilmann RM, Campbell SM, Kroner BA et al. Evolution, current structure, and role of a primary care clinical pharmacy service in an integrated managed care organization. Ann Pharmacother. 2013;47:124-31.

63. Reta A, Dashtaei A, Lim S et al. Opportunities to improve clinical outcomes and challenges to implementing clinical pharmacists into health care teams. Prim Care. 2012;39:615-26.

64. Bodenheimer T. Coordinating care - a perilous journey through the health care system. $N$ Engl J Med. 2008;358:1064-71.

65. Billups SJ, Delate T, Newlon $\mathrm{C}$ et al. Outcomes of a pharmacist-managed medication refill program. J Am Pharm Assoc. 2013;53:505-12. 
66. Stubbings T, Miller C, Humphries TL et al. Telepharmacy in a health maintenance organization. Am J Health-Syst Pharm. 2005;62:406-10.

67. American Association of Medical Colleges. The road to becoming a doctor. https://www.aamc.org/download/68806/data/ (accessed $2013 \mathrm{Jul} 28$ ).

68. Osborne MA, Snyder ME, Hall DL et al. Evaluating Pennsylvania pharmacists' provision of community-based patient care services to develop a statewide practice network. Innov Pharm. 2011;4:1-8.

69. Anon. California provider status bill passes state legislature, headed to governor. http://www.pharmacist.com/california-provider-status-bill-passes-state-legislature-headedgovernor (accessed 2013 Sep 27).

70. Thompson CA. California bill would accord pharmacists provider status. Am J Health-Syst Pharm. 2013;70:839-40.

71. Look KA, Mott DA, Leedham RK et al. Pharmacy participation and claim characteristics in the Wisconsin Medicaid harmaceutical care program from 1996 to 2007. J Manag Care Pharm. 2012;18:116-28.

72. Michaels NM, Jenkins GF, Pruss DL et al. Retrospective analysis of community pharmacists' recommendations in the North Carolina Medicaid medication therapy management program. J Am Pharm Assoc. 2010;50:347-53.

73. Carmichael JM. Pharmacy's bucket list: lean in. Am J Health-Syst Pharm. 2013;70:1375-82.

74. Helling DK. Lengthen your stride. J Am Pharm Assoc. 2013;53:324-26. 